\title{
USO Y MANEJO DE LEÑA POR LA COMUNIDAD CAMPESINA DE SAN JOSÉ DE SUAITA (SUAITA, SANTANDER, COLOMBIA)
}

Palabras clave: Andes, comunidades campesinas, etnobotánica, leña, uso y manejo.

Key Words: Andes, rural communities, ethnobotany, firewood, use and management.

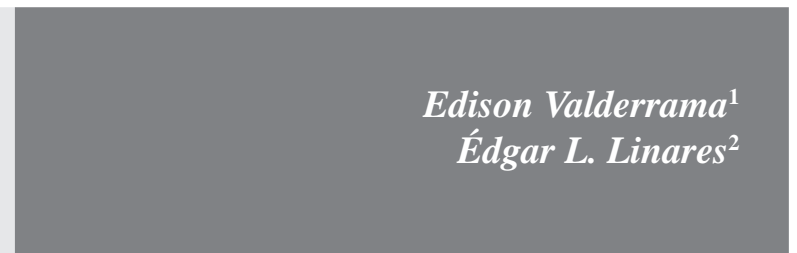

\section{RESUMEN}

La utilización de leña por parte de las comunidades campesinas de los Andes de Colombia siempre ha sido vista como una práctica cultural que afecta negativamente los bosques silvestres cordilleranos. En la Inspección de Policía de San José de Suaita, mediante el uso de encuestas semiestructuradas, se evaluó el uso y manejo de leña por parte de habitantes del campo y del casco semiurbano. Los resultados muestran prácticas culturales que reflejan estrechas relaciones con el medio circundante: conocimiento de la dinámica de regeneración de la vegetación, utilización de diversos sistemas agrícolas como fuente de leña, utilización amplia de taxonomías populares para distinguir cada especie y valoración de la calidad de la leña.

\section{ABSTRACT \\ The firewood use on the part of the rural communi- ties of the Colombian Andes has always been seen as a cultural practice that affects the mountain wild forests negatively. In San José of Suaita's Police Inspection, firewood use and handling on the part of inhabitants of the field and of a semi-urban hel- met. The results show cultural practices that reflect interesting relationships with the surroundings: knowledge of the dynamics of regeneration of the vegetation, use of diverse agricultural systems as}

firewood source, wide use of popular taxonomies to distinguish each species and valuation of the quality of the firewood.

\section{INTRODUCCIÓN}

Los bosques neotropicales montanos están entre los menos conocidos y más amenazados de todas las formaciones tropicales (Gentry 2001). En Colombia diferentes estimaciones sugieren que quedan menos del 10\% de los bosques andinos (Henderson et al. 1991) y en la actualidad se considera a los bosques premontanos o subandinos como uno de los ecosistemas más amenazados del país. Las áreas de bosque subandino $(1.000-2.400 \mathrm{~m})$ son cada vez más reducidas en Colombia, debido entre otras razones a la ampliación de zonas de cultivo y a las necesidades de madera y leña por parte de las comunidades campesinas, porque no existen alternativas energéticas para la leña.

A finales de los años 80, Colombia tenía un remanente de bosque de 53.1 millones de hectáreas y su deforestación se estimaba en unas 600.000 ha/año debido a la colonización agrícola (76.3\%), al consumo de combustible $(12.7 \%)$ y a las necesidades de maderas industriales (11\%) (DNP 1989). Recientemente se estimó que en el año 2000 la leña tuvo un papel preponderante en el consumo energético con una participación del $40 \%$ del total nacional

1 Corporación para la Conservación, Investigación y Educación Ambiental desde la Etnobiología, Corporación Tanta, edvalder@hotmail.com.

2 Instituto de Ciencias Naturales, Facultad de Ciencias, Universidad Nacional de Colombia. A.A. 7495, Bogotá, Colombia, ellinaresc@unal. edu.co. 
(incluyendo el sector urbano) y del $80 \%$ en el sector rural (SIAC 2002). Según la misma fuente el aumento de la demanda de leña puede ocasionar problemas de deforestación, pero por otro lado si el recurso forestal fuera aprovechado sosteniblemente podría ser una fuente de energía continua.

San José de Suaita, Inspección de Policía del municipio de Suaita en el departamento de Santander, posee algunos relictos del bosque subandino original -específicamente en la zona conocida como "La Meseta"-, que son el eje ambiental de la región, gracias a que proporcionan agua para algunas veredas de los municipios de Suaita y Guadalupe, por lo que constituyen una reserva de servicios ambientales para los habitantes de las áreas rurales.

En una fase previa de aproximación a la comunidad, mediante un "Taller de identificación de problemáticas ambientales", la comunidad campesina de San José de Suaita identificó, dentro de una serie de problemáticas, la tala del bosque (deforestación) y quema de rastrojos como dos de los problemas ambientales más importantes; así mismo identificaron sus causas: necesidad de madera para construcción, necesidad de leña para cocinar, despejar áreas para cultivos (caña, maíz, y yuca) y ganadería, "por costumbre" y para cubrir necesidades económicas. Esto ha generado otras consecuencias que igualmente fueron identificadas por la comunidad: pérdida de la fertilidad y de los microorganismos del suelo, erosión, escasez de agua, pérdida de plantas de importancia cultural, además de pérdida de la biodiversidad.

En la cuenca del río Suárez (región a la que pertenece el área de estudio) existen costumbres culturales sobre la utilización de la leña que se obtiene de áreas ambientalmente importantes como bosques naturales, bosques secundarios, áreas de cultivo y en barbecho, potreros y márgenes de quebradas, arroyos y caños. Algunas veces la leña es extraída como ramas caídas de los árboles durante la época seca, de la tala de árboles, u obtenida de especies agronómicamente importantes como el "guamo" (Inga spp.) que sirven de sombrío a cultivos como el café, o en algunos casos es comprada a personas externas a la comunidad. Este artículo es una aproximación a la forma en la que el recurso leña es aprovechado por una comunidad campesina colombiana, evaluando el consumo, la forma de recolección y las especies utilizadas.

\section{ÁREA DE ESTUDIO}

El estudio se llevó a cabo en la Inspección de Policía de San José de Suaita, perteneciente al municipio de Suaita (departamento de Santander), situado sobre la Cordillera Oriental (Andes colombianos), en la unidad fisiográfica de los valles longitudinales de los ríos Fonce y Suárez, formada por valles profundos encajonados y mesetas escalonadas (IGAC 1982), con alturas entre los 1.500 y $2.100 \mathrm{~m}$. Con un área total de 4.846 ha, el municipio de Suaita tiene una temperatura media de $19^{\circ} \mathrm{C}$ y una precipitación media de 2.719,7 m anuales para la estación de Cielo Roto $\left(06^{\circ} 07^{\prime} \mathrm{N}\right.$ y $\left.73^{\circ} 28^{\prime} \mathrm{W}\right)$. Como consecuencia de su posición en la zona de confluencia intertropical, la precipitación es de distribución bimodal; de abril a mayo es la primera temporada de lluvias del año y de octubre a noviembre la segunda, siendo octubre el mes más lluvioso (IDEAM 1998); la primera temporada seca ocurre entre diciembre y enero y la segunda entre julio y agosto.

Según Holdridge (1977), el área de estudio corresponde a la formación vegetal bosque muy húmedo premontano (bmh-PM), de la cordillera Oriental. En un inventario previo, las familias de dicotiledóneas más diversas son, en su orden: Melastomataceae (26 especies), Rubiaceae (23 especies), Asteraceae (13 especies), Piperaceae (13 especies), Euphorbiaceae (11 especies), y Myrtaceae (10 especies). En cuanto a monocotiledóneas las más importantes son: Orchidaceae (72 especies), Bromeliaceae (37 especies), Poaceae (20 especies), Araceae (16 especies) y Arecaceae (10 especies) (Murillo et al. 2002).

La Inspección cuenta actualmente con 203 unidades familiares y 639 habitantes, la mayor parte personas en edades jóvenes (Figura 1). El 47.7\% de la población no tiene ningún nivel educativo, el $40.4 \%$ de la población tiene algún nivel en educación básica primaria, mientras que el $1.4 \%$ y el $0.5 \%$ tiene algún nivel en educación media y universitaria, respectivamente (datos facilitados por la parroquia de San José). 


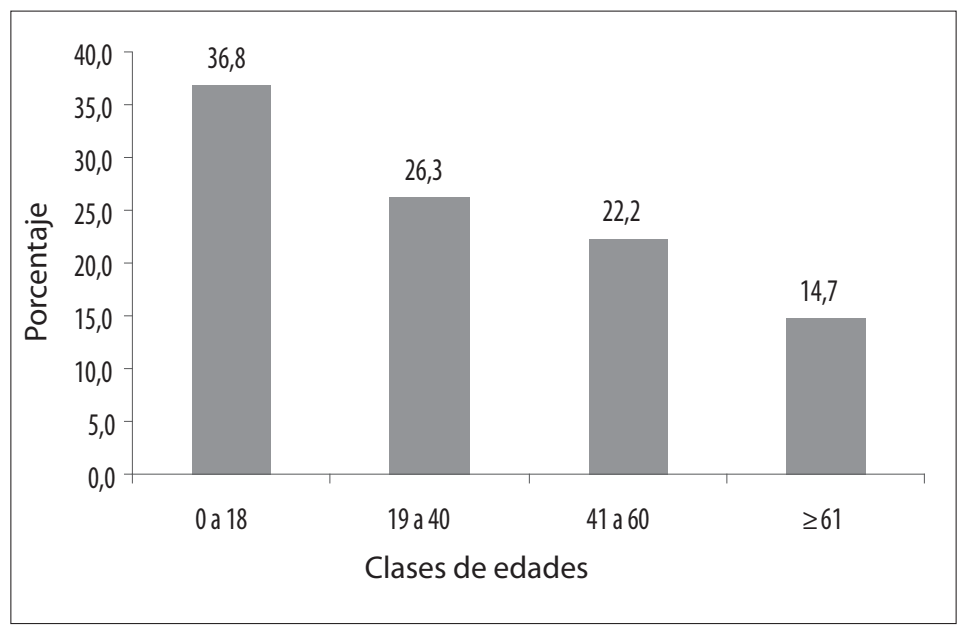

Figura 1. Distribución de edades de los habitantes en San José de Suaita (a partir de datos suministrados por la parroquia de San José).

En cuanto a la tenencia de la tierra, según el ICA (en Agamez 1997), en el municipio de Suaita de los 2.333 predios totales, el $65.3 \%$ son minifundios (0,5-5 ha; $8.61 \%$ de la superficie), ubicados en su mayoría en zonas de ladera, el $23.9 \%$ de los predios son pequeñas propiedades entre 5.1-10 ha (19.94\% de la superficie), el $5.9 \%$ pertenecen a la categoría Unidad Familiar con fincas de 10.1-20 ha (14.98\% de la superficie), las medianas propiedades (20.150 ha) son el $2.6 \%$ de los predios $(15.21 \%$ de la superficie) y, por último, el $2.31 \%$ de los predios son latifundios ( $>50$ ha; $41.26 \%$ de la superficie).

\section{MÉTODOS}

Previo al presente estudio se hizo una encuesta estructurada con la cual se entrevistaron 98 hogares (representatividad del 48\%), en la que se evaluó el tamaño del núcleo familiar, el acceso a otras fuentes o servicios de energía, el acceso al transporte, la distancia al lugar de recolección, el consumo diario y la frecuencia diaria de utilización de la leña. Para los análisis estadísticos, se usaron pruebas no paramétricas de Kruskall-Wallis, con un nivel de confianza $\alpha=0.05$, utilizando el software StatGraphics Plus 4.0.

De los 98 hogares, se seleccionaron cinco viviendas del sector rural en las que mediante observación participante en cada una, durante una semana se hizo seguimiento al consumo de cada familia.
Haciendo entrevistas semiestructuradas y abiertas (Alexiades 1996, Martin 2000) dirigidas a quienes utilizan la leña en estas familias (como las mujeres encargadas de la cocina), se establecieron los usos dados a ésta, así como criterios de calidad y tiempos de preparación de alimentos. También se hicieron entrevistas a recolectores de estas familias sobre las especies utilizadas, formas y sitios de recolección, además el tiempo de recolección (en horas), distancia a la fuente, consumo y tiempos de combustión diarios y semanales.

En caminatas botánicas, junto a los recolectores de las cinco familias se identificaron las especies utilizadas y a través de observación participante se complementó la información relacionada sobre formas de recolección y partes cosechadas (ramas, tronco y raíz), tiempos y distancias empleadas y las fuentes productoras de leña (especies y sus hábitats). De los ejemplares recolectados se recogió información sobre localidad, nombre local de la planta, hábitat, hábito, altura, DAP, color de la flor y del fruto, partes utilizadas y otros usos. Las colecciones fueron depositadas en el Herbario Nacional Colombiano (COL), del Instituto de Ciencias Naturales de la Universidad Nacional de Colombia.

Con la información obtenida de las encuestas semiestructuradas se elaboró una base de datos a partir de la cual se analizaron variables cuantitativas sobre la forma de recolección en cada familia. Además, se 
analizaron las variables cualitativas como la especificidad de uso por especie y la calidad de la leña. Para evaluar el consumo se tomó en cuenta la cantidad de leña necesaria para obtener la energía indispensable para el consumo familiar doméstico por semana. Ésta se midió pesando un lote de leña antes de cada día y pesando la leña restante al final del día. También se evaluó el consumo total por especie.

\section{RESULTADOS}

\section{CONSUMO DE LEÑA}

El estudio fue enfocado principalmente en el nivel residencial representado en la Unidad de Producción Campesina (sector rural). Los resultados presentados a continuación son expresados como descripción de las formas de apropiación, obtención y consumo de leña en el área rural, aunque también se hacen algunas anotaciones sobre el casco urbano (caserío) de la Inspección de Policía que, al igual que las familias rurales, presenta similares modelos de uso y manejo de leña, por estar enclavado en un área rural.

El hogar promedio en San José de Suaita consta de tres personas (Figura 2). El $34.4 \%$ de la población son mujeres adultas, el $34.4 \%$ son hombres adultos y el $31.2 \%$ son niños. El número de personas por hogar tiene incidencia significativa sobre el consumo $(\mathrm{H}=15,9738$; $\mathrm{p}$-valor $<0.05)$ y en el diagrama de box-and-whisker el comportamiento de las medias refleja aumento del consumo de leña a medida que aumenta el número de personas. Las cajas de las categorías más bajas $(1,2$ y 3$)$ muestran una amplia distribución de los datos reflejando que estas familias tienen tendencia hacia consumos altos, similares a familias con mayor número de personas, cuyas cajas muestran una distribución más homogénea (4, 5 y 6) (Figura 3).

El 52,2\% de los hogares del sector rural y el $85,2 \%$ del sector urbano reconocieron utilizar gas como fuente alternativa de energía para cocinar. No obstante, el $98.6 \%$ de las viviendas del sector rural y el $81.5 \%$ del sector urbano emplearon leña como fuente de energía para cocinar. En cuanto al acceso o no al gas como fuente alternativa de energía, no hay diferencia estadísticamente significativa con relación al consumo de leña $(H=1,80777$; $p$-valor $>0.05)$.

El 52\% de la población rural transportaba la leña a pie, mientras que el $42 \%$ utilizaba caballos o mulas y el $4,4 \%$ poseía un vehículo. En cuanto a las distancias recorridas a la fuente de obtención de leña, el $80 \%$ de los hogares recolectaba leña a menos de $1 \mathrm{~km}$ de distancia de su casa, mientras que el 20\% lo hacía a una distancia mayor a $1 \mathrm{~km}$. En las cinco viviendas visitadas, la mayoría de veces la leña se conseguía a distancias entre los 100 y 300 m a

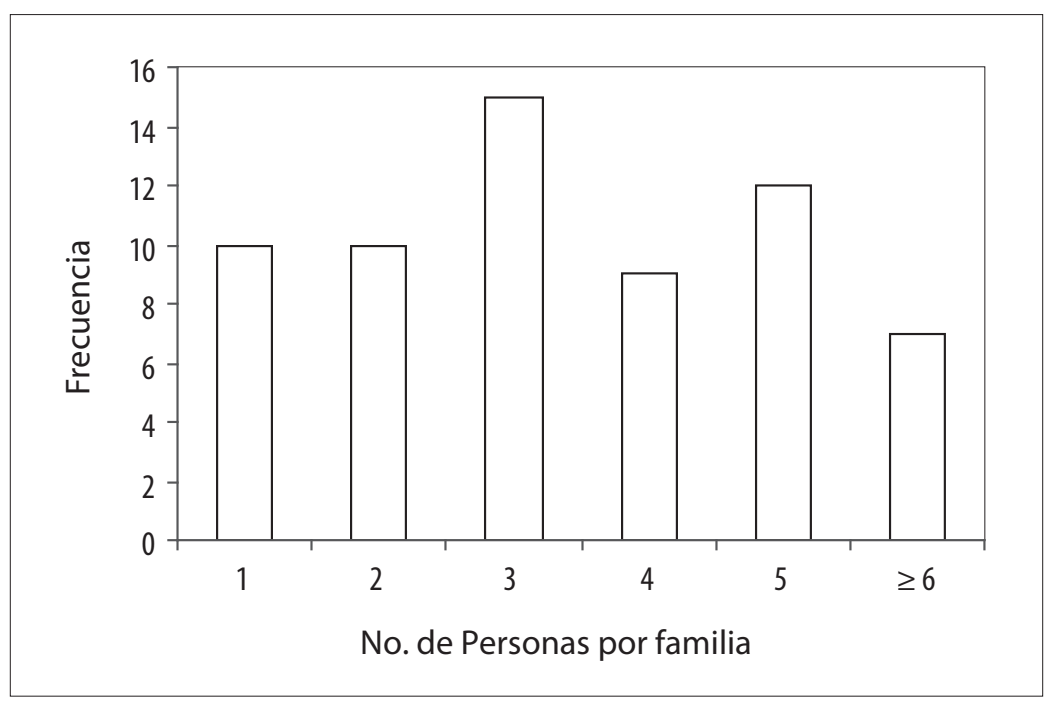

Figura 2. Distribución de frecuencias del $\mathrm{N}^{\mathrm{o}}$ de personas por familias. 


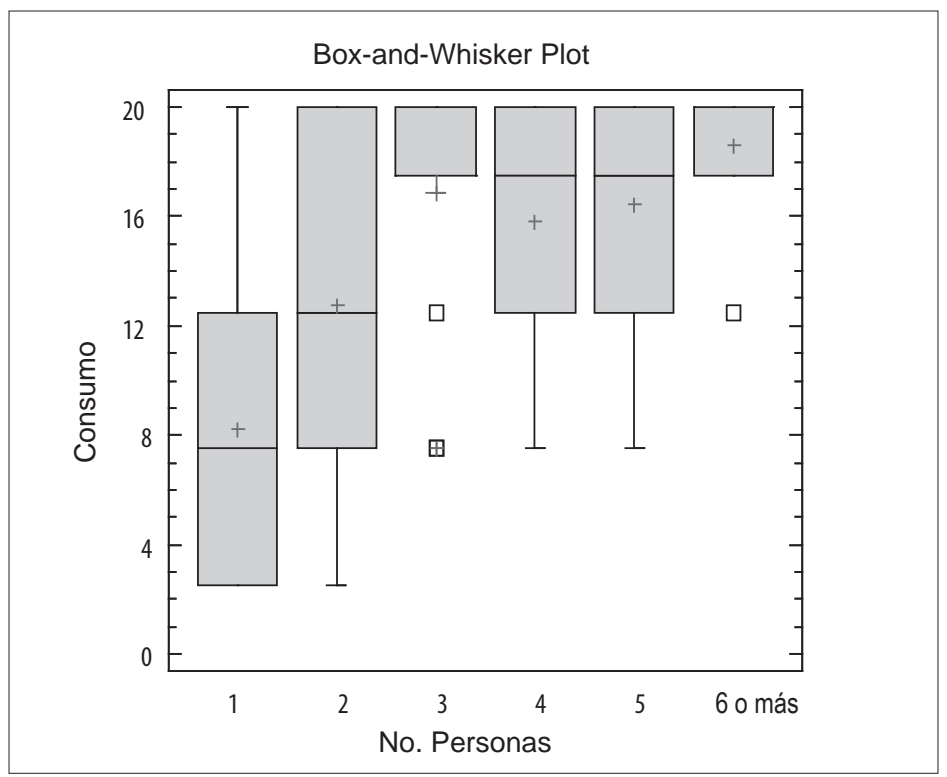

Figura 3. Análisis de box and whisker plot que relaciona al $\mathrm{N}^{\circ}$ de personas por familia con el consumo de leña.

pie, debido a que la leña se encontraba disponible dentro de la misma finca y era suficiente para satisfacer las necesidades energéticas de la familia. No obstante, hay algunas familias que tenían que desplazarse más de $400 \mathrm{~m}$, pues no se encontraba el recurso dentro de la finca, ni en las de vecinos cercanos. Según la opinión de los recolectores ellos no notaban diferencias en las distancias recorridas hace 20 o 30 años con relación al presente, por lo que se asume que las personas no perciben que haya una escasez de leña en San José, ya que consideran que se consigue fácilmente. Ni en la disponibilidad de transporte $(\mathrm{H}=1,80777$; $\mathrm{p}$-valor $>0.05)$, ni en la distancia a recorrer $(\mathrm{H}=1,80777$; p-valor $>0.05)$, se muestran diferencias estadísticamente significativas frente al consumo.

Tanto el hombre como la mujer participan de las labores de recolección de leña, sin embargo la mujer se encarga de la administración y uso de la misma. Son las mujeres las encargadas de la preparación de las comidas para el hogar y para los obreros que colaboran en las labores de la finca. Al obrero (jornalero) como parte de pago se le dan 4 comidas o "costos" (6 en el caso de la molienda para producir panela) durante el día: desayuno, puntal, almuerzo y comida, hecho que puede aumentar drásticamente el consumo de leña. También se preparan alimentos para los animales como pollos, cerdos y cabras, entre otros.

Los alimentos más empleados en la cocción son principalmente tubérculos y cormos como el bore, la yuca, la papa (no cultivada en la región) y el chonque, cereales como maíz y arroz y legumbres como el fríjol; además son muy utilizados en la dieta el plátano y productos de origen animal como la carne de res, cerdo, pollo (gallina) y carne de monte producto de la cacería: chucha o fara, perezoso, armadillo y picure, entre otros. De éstos el bore, la yuca y el maíz son los más empleados y su consumo en grandes cantidades hace que el gasto de leña aumente considerablemente en los hogares donde se preparen.

El 58\% de las familias del sector rural mencionaron prender el fogón 3 veces al día, mientras que el $23.5 \%$, lo hacen 4 o más veces en el día y de éstos algunos lo dejan encendido todo el día; únicamente el $17.6 \%$ utiliza el fogón menos de tres veces al día; sin embargo esto puede variar según la época del año por las actividades agrícolas que se estén realizando en ese momento en la finca y por consiguiente la presencia de obreros puede aumentar el número de veces que se prende el fogón en el día. El número de veces que se prende el fogón en el día tiene relación estadísticamente significativa 
Tabla 1. Consumo semanal de leña en cinco familias.

\begin{tabular}{ccccccc}
\hline Familia & Consumo $(\mathrm{kg})$ & Per cápita & Miembros & Hectáreas & Fogón & \multicolumn{2}{c}{ Horas de combustión/ } \\
Semana
\end{tabular}

$(\mathrm{H}=11,2604$; p-valor $<0.05)$ con el consumo. El tiempo promedio de cocción es de 7 horas, de las cuales entre las horas de la mañana (entre 4 y $10 \mathrm{am}$ ) y la tarde (entre 2 y $8 \mathrm{pm}$ ), se mantiene prendido el fogón entre 2.7 h y 2.3 h respectivamente; mientras que al medio día (10 am a 2 pm) el fogón se prende durante 2 horas en promedio.

En San José de Suaita se utilizan dos tipos de fogón, uno cerrado (52.2\%), que consiste en una estufa con paredes en ladrillo que es alimentada con leña o carbón y que controla el flujo de calor al exterior, y otro abierto (47.8\%), que a diferencia del cerrado no tiene paredes y está expuesto al medio, por lo tanto no se controla el flujo de calor hacia el exterior. Esta variable no incide significativamente en el consumo de leña $(H=0,225015$; $p$-valor > $0.05)$, sin embargo de las cinco viviendas a las que se hizo seguimiento, aquellas donde se cocina con fogón abierto son mucho más consumidoras que las que usan fogón cerrado (Tabla 1).

Basado en el seguimiento semanal a cinco familias, el consumo promedio por familia en San José de Suaita fue de $157.5 \mathrm{~kg}$ en una semana, y $4.8 \mathrm{~kg} /$ cápita/día (Tabla 1). El consumo anual por familia es de 8,212 t. Estas cifras son bastante altas si se compara con las obtenidas en X-Uilub (México) de 4,193 t/año a nivel familiar y de 2.02 kg/cápita/día (Sánchez 1993).

\section{FUENTES DEL RECURSO}

Los campesinos obtienen la leña de diversas fuentes. Según la encuesta, el 33.8\% de los hogares obtienen la leña de una sola fuente, mientras que el $42.6 \%$ y el $22.1 \%$ la obtienen de dos y tres fuentes respectivamente y tan solo el $1.5 \%$ la logran de más de 3 fuentes. El cultivo de café es la principal fuente de obtención de leña en el sector rural, pues el 58.8\% de las personas dicen obtener leña de éste, mientras que el $41.2 \%$ no. En segundo renglón, los residuos de labranza con un $36.8 \%$ de la población dice utilizarlos, contra un $63.2 \%$ que no; la leña seca lograda de diversas fuentes es empleada por el $41.2 \%$ de las viviendas contra un $58.8 \%$ que no la utiliza (Figura 4 ).

Otra fuente importante son las áreas de bosque secundario mayores de 10 años definidas como "varsales", siendo empleadas para la obtención de leña por un $29.4 \%$ de la población rural, mientras que el 70,6\% manifestó no utilizarlos con este fin. El $13.2 \%$ afirmó utilizar las áreas de bosque poco intervenido o en regeneración, conocidos como "rastrojeras", para obtener leña, en tanto que el $86.8 \%$ afirmó no hacerlo. Por último el $11.8 \%$ del total de viviendas compra la leña a otras personas (asociado al tipo de recolección III, (Tabla 4). En cuanto a las áreas pertenecientes a los márgenes de quebradas, la comunidad entrevistada manifestó no realizar extracción de leña en estos sitios, excepto la que arrastran las quebradas en la época invernal y que es recogida por ellos. En el sector urbano la leña comprada es la principal fuente de obtención del recurso, en el $54.5 \%$ de los hogares, mientras que en el $45.5 \%$ no; por otro lado el $36.4 \%$ de los hogares obtienen leña de recolección de palos caídos y secos, ya sea en su propia finca o en otras partes. La leña comprada se obtiene principalmente de bosques secundarios, localmente conocidos como "rastrojeras". 


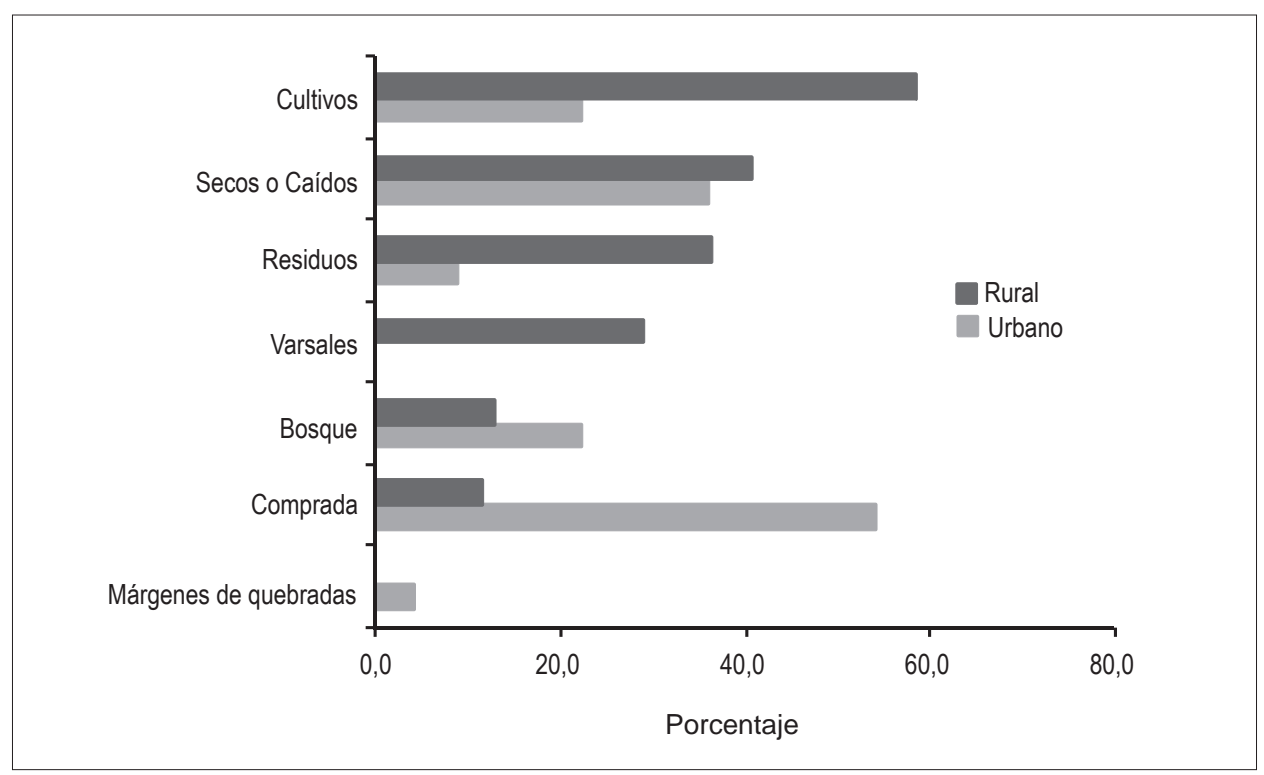

Figura 4. Porcentaje de las fuentes de obtención de leña, según la población rural de San José de Suaita.

\section{ESPECIES}

Se encontraron un total de 69 especies vegetales que fueron reportadas por la comunidad con uso combustible, de las cuales 52 efectivamente son utilizadas como leña, ya que se observó su uso por lo menos una vez (Anexo 1). Estas 52 especies están distribuidas en 27 familias, de las cuales las más importantes fueron Melastomataceae ( 9 spp.), Myrtaceae (5 spp.), Euphorbiaceae (5 spp.) y
Mimosaceae (4 spp.). En cuanto a las restantes 17 especies, de las que no se observó uso como leña, la comunidad afirmó haberlas utilizado alguna vez con este fin. Según su forma de vida, el $86.3 \%$ son árboles y el $13.7 \%$ son arbustos.

De acuerdo a la clasificación de las especies según su intensidad de uso (Tabla 2), se aprecia cómo los niveles más altos de consumo están concentrados en

Tabla 2. Porcentaje de consumo de las especies más utilizadas como leña en San José de Suaita.

\begin{tabular}{llc}
\hline Nombre científico & Nombre local & \% del total consumido \\
\hline Miconia dolichorrhyncha & Macanillo esmeraldo, Macanillo blanco & 20.8 \\
Inga aff. ingoides, I. spectabilis**, I. oerstidiana & Guamo* & 14.8 \\
Coffea arabica var. Colombia & Café & 6.7 \\
Miconia minutiflora ${ }^{*}$, M. cremophylla & Tuno blanco* & 6.7 \\
Myrcia aff. fallax & Payo & 6.1 \\
Vismia sp. 2 & Manchador & 5.1 \\
Miconia serrulata, M. Elata & Macanillo negro* & 5.0 \\
Henriettella seemannii & Cubo'y hierro & 4.3 \\
Ficus sp. & Lechero 0 Chivechi & 2.7 \\
Demás especies (38) & & 27.8 \\
\end{tabular}

* Incluye el valor para más de una especie con el mismo nombre local.

**Especie más consumida. 
13 especies que constituyen el $72.2 \%$ del consumo total, mientras que las demás especies (38) de las clases medias y bajas corresponden al $27.8 \%$. De estas especies con alto nivel de consumo, 4 de ellas hacen parte del $35.6 \%$ del consumo total y Miconia dolichorrhyncha soporta el $20.8 \%$ del consumo total. Las otras tres son especies de guamo (Inga spp.), las cuales cumplen además la función de sombrío para los cultivos de café. La mayoría de especies tienen muy bajo y bajo nivel de consumo (29), disminuyendo en las clases intermedias y altas (10 y 9 respectivamente) y 4 especies en el nivel más alto (véase Anexo 1).

\section{HÁBITATS}

El 94\% de las especies están en uno o varios de los agroecosistemas manejados por el campesino, tales como rastrojeras y varsales (bosques secundarios o en regeneración), cafetales, potreros o en bordes de camino; en tanto que tan sólo el 6\% de las especies son exclusivas del bosque primario.

Tabla 3. Número de especies utilizadas como leña, por hábitat.

\begin{tabular}{lc}
\hline Hábitat & No de especies $^{\circ}$ \\
\hline Bosque en regeneración "rastrojera" & 25 \\
Bordes de camino & 24 \\
Cafetales & 16 \\
Potreros & 10 \\
Barbechos "varsal" & 9 \\
Bosque poco intervenido & 3 \\
\hline
\end{tabular}

Las rastrojeras o bosques en regeneración concentran el mayor número de especies utilizadas como leña, con 25 , y los bordes de camino o carreteras con 24 especies, los cafetales con 16, y los potreros y varsales con 10 y 9 especies, respectivamente (Tabla 3). Hay una gran cantidad de especies que están presentes en áreas muy deterioradas como las que han sido destinadas a potreros y los sectores que rodean y delimitan la avanzada de los tipos de vegetación encontrados (rastrojera y varsal).

\section{FORMAS DE APROPIACIÓN DEL RECURSO}

La Tabla 4 muestra los tipos de apropiación de leña más importantes, según las indicaciones de los recolectores y la observación participante. Su frecuencia e intensidad de recolección están condicionadas por la composición de los agroecosistemas y la temporalidad durante el ciclo de producción anual. De este modo tenemos el sistema de Roza, tumba y quema para las labranzas (Tipo I), Recolección de palos secos o caídos, que a su vez difiere según su destino en autoconsumo (Tipo II) o venta (Tipo III).

\section{TUMBA, ROZA Y QUEMA (TIPO I)}

Este tipo de recolección se hace entre el final y el comienzo de año (diciembre-marzo), que coincide con la época seca. Durante este tiempo se realizan actividades agrícolas importantes que determinan el ciclo de producción anual. A estas actividades se las conoce con el nombre de labranza e incluye las labores de rocería, siembra, limpieza y cosecha de los cultivos. Una vez establecido el sitio en el cual se va a sembrar, se hace la primera actividad que es la tumba, roza y quema del terreno; esta práctica de quema es muy generalizada especialmente en cultivos de cielo abierto como la caña panelera, maíz y yuca, que requieren eliminar árboles que puedan darle sombra al cultivo. Otros cultivos como el café y el plátano no requieren de una tumba excesiva y permiten que se conserven algunos árboles con varios propósitos como dar sombra y para el consumo de leña. Este proceso es realizado por los hombres, ya sean obreros o propietarios de la tierra.

Tabla 4. Caracterización de las formas de apropiación de leña.

\begin{tabular}{lllll}
\hline Tipo de recolección & Nivel de extracción & Destino & Frecuencia de recolección & Zonas de extracción \\
\hline I & Alto & Vivienda - Venta & Anual - Semestral & Varsal - Cafetal \\
II & Bajo & Vivienda rural & Diario y día por medio & Varsal - Cafetal \\
III & Medio & Venta - Urbano & Semanal - Quincenal & Rastrojera - Varsal \\
\hline
\end{tabular}


$\mathrm{Su}$ frecuencia de extracción es anual a semestral y la leña obtenida es destinada para el consumo en las viviendas y la agroindustria (molinos paneleros) y algunas veces se destina para la venta. De esta actividad se obtiene gran cantidad de leña que es apilada cerca de la casa o de los caminos; estos grandes almacenamientos de leña se denominan "marcos" y pueden llegar a tener entre 250 (el equivalente a 1 ha de cafetal) a 3.000 cargas, según el tamaño del área tumbada, el tipo de cultivo o el tiempo de abandono del barbecho. La disposición de los "marcos" depende de la distancia de la parcela a la carretera, algunas veces la leña no puede ser sacada en su totalidad y queda dentro del cultivo, por lo que es regalada o vendida al aparcero o a los vecinos pero con la condición de que ellos deben ir a buscarla. Cuando la leña se junta al borde de la carretera, es destinada para el autoconsumo de la finca o para la venta a los molinos paneleros.

Toda esta actividad se realiza en tres etapas:

Macaneo o "rocería”: en la que cuadrillas de obreros cortan con machete o guadaña, a ras del suelo, toda la vegetación herbácea y arbustiva. Durante esta práctica algunos campesinos dejan en pie individuos vivos o tocones de árboles útiles como: amarillo mango (indet.), arrayán (Calycolpus moritzianus), galapo (Albizia carbonaria), guamo (Inga cf. ingoides, I. spectabilis e I. oerstidiana), manchador (Vismia sp.1), menudito (Psidium sp.), macanillo esmeraldo (Miconia dolichorrhyncha), macanillo negro ( $M$. serrulata) y payo (Myrcia aff. fallax). La intensidad de esta labor depende del tipo de cultivo que se planee sembrar, de esta manera cuando el terreno se va a destinar a caña panelera no se deja ningún tipo de árbol, si su destino es la siembra de yuca, maíz o fríjol, se suelen dejar algunos árboles como el soque (Acacia sp.), el balso (Heliocarpus americanus) o el ortigo; de éstos únicamente el soque es reportado en este estudio con uso de leña. Si el cultivo a sembrar es de café se dejan árboles como galapo, guamo, macanillo esmeraldo y macanillo negro. Cuando se pretende abrir un área de potrero las personas acostumbran dejar crecer individuos de arrayán, menudito, macanillos esmeraldo y negro, guayabo, payo y tuno blanco (Miconia minutiflora), preferiblemente como cercas vivas o dentro del potrero pero con una distribución espacial muy amplia.

Tumba: se hace sólo cuando hay árboles muy grandes que no pueden ser tumbados con machete, y para ello se emplea hacha o motosierra dependiendo del tamaño del área a "despejar".

Quema: según el concepto de los campesinos ésta se hace con el fin de incorporar rápidamente nutrientes al suelo y mejorar su fertilidad, además de economizar (en cuanto al pago de obreros) la limpieza del terreno antes de la siembra. Se hace al final de la época de sequía cuando el material vegetal se encuentra totalmente seco. Algunos de los entrevistados no están de acuerdo con esta práctica, pues consideran que empobrece el suelo y en lugar de fertilizarlo lo vuelve cada vez más estéril.

\section{RECOLECCIÓN DE PALOS SECOS O CAÍDOS (TIPO II)}

Se hace durante todo el año, lo que la convierte en una búsqueda diaria. Esta forma de recolección se hace a pie y por lo tanto sólo permite traer una "brazada" a la vez. Una brazada de leña tiene de 20 a 25 kilogramos y está compuesta de ramas delgadas. De esta manera hombres, mujeres y jóvenes mayores contribuyen en la apropiación de leña.

La recolección se hace manualmente, en el caso de palos y ramas secas caídas y con machete o hacha para árboles y tocones secos en pie. En la mayoría de casos se hace en los cultivos o labranzas cercanas que ya han pasado por el proceso de roza, tumba y quema, en las áreas de cultivo que han sido abandonadas (varsales) y a partir de los árboles de sombrío del cafetal (Tabla 1). También se hace en bordes de caminos, en las rastrojeras, en potreros, en las cercas y en las riberas de los ríos. Son aprovechados bajo esta categoría los excedentes de la tala de árboles para madera, los "morones" o postes de cerca fabricados con especies como macanillo blanco, macanillo negro, arrayán, guamo, bailador y tuno blanco. Otra práctica es la de hacer un anillo a la corteza del árbol y dejarlo secar para luego cosecharlo a medida que se va necesitando, especialmente en árboles con copas abiertas que 
forman muchas ramas como el galapo, el guamo y los macanillos.

\section{RECOLECCIÓN DE PALOS SECOS Y CAÍDOS PARA LA VENTA (TIPO III)}

Este método de recolección es similar al Tipo II, pero con la diferencia de que se hace en mayor cantidad ya que se emplea un animal para transportarlo y por la misma razón se hace en sitios más alejados como las rastrojeras y varsales con más de 20 años de abandono, de donde se puede obtener "buena leña". La cantidad extraída por este método puede ser de una a dos cargas (250 kg aproximadamente) y está destinada por lo general a la venta, en su mayoría en el casco urbano de San José de Suaita y también para el autoconsumo. En este tipo de recolección se selecciona la leña más gruesa y pesada o de mejor calidad para la combustión con destino a la venta y dejan la más liviana y delgada para el autoconsumo. En este método se observó que algunos de los palos recolectados han sido previamente tumbados y dejados en el sitio hasta secarse (aproximadamente 20 días), al cabo de lo cual se pican y se transportan a su destino.

\section{CONOCIMIENTO DEL CAMPESINO SOBRE LOS USOS DE LA LEÑA}

El campesino en San José de Suaita reconoce cuándo un "palo" es bueno para leña y cuándo debe usarlo; en este sentido, la sociedad campesina tiene capacidad para clasificar cualitativamente la calidad de la leña respecto de su utilidad para producir fuego. En cuanto a los usos, aunque prácticamente todos los tipos de leña son usados en la preparación de alimentos, hay algunos usos específicos identificados: para el encendido del fogón (mina), por su rapidez de combustión, utilizan mucho cáscaras de fríjol y maíz, hojas secas de plátano, quiches (Bromeliaceae) y varas delgadas o astillas de algunas especies, entre las que se destacan la guadua (Guadua angustifolia), por su facilidad para hacer astillas) y el cucharo (Clusia sp.), así como el bagazo seco de la caña panelera (Saccharum officinarum). Para "guardar la candela" (conservar la brasa) es preferida la madera de guamo, gaque (Clusia sp.) y pomarroso (Syzygium jambos), aunque de esta última no se registró consumo. En cuanto a la producción de ceniza, cuyo uso es excepcional, ya que sólo algunos la usan, revuelta con gallinaza, como correctivo de los cultivos o de las huertas, sólo se reporta el balso (Heliocarpus americanus), ya que "forma gran cantidad de ceniza en poco tiempo".

La calidad de leña es relativa para el campesino, pues para algunos "cualquier palo sirve como leña siempre y cuando prenda", y no se encontraron categorías de calidad para ésta, sin embargo las especies consideradas buenas son aquellas que al prender el fogón pueden guardar mejor el calor (rendimiento de la leña) y "no se consumen rápidamente", de tal manera que alcanzan para la preparación de una o varias comidas. También son consideradas buenas o excelentes por su mayor peso (estas especies pueden tener altas densidades debido a una mayor relación peso/volumen y realmente su tiempo de combustión es mayor al de otras maderas de menor densidad), ya que estas maderas "prenden" mejor, a diferencia del balso y el lechero, considerados de baja calidad de combustión porque se dice que la madera es "cenizosa", es decir que se consume o quema muy rápido y "no hace brasa". Muchas de las especies consideradas excelentes como leña fueron las más consumidas, como el macanillo esmeraldo (Miconia dolichorrhyncha) y los guamos (Inga spp.), sin embargo el arrayán (Calycolpus moritzianus) es poco utilizado por cuanto es difícil de conseguir. El gaque se reconoce como una especie que puede "reventar" durante su combustión y no es recomendada para la ignición; finalmente, el cedro (Cedrela odorata), a pesar de ser considerado como buena madera, no se considera buena leña sino más bien "cenizosa".

\section{DISCUSIÓN}

El consumo de leña estimado en $4.8 \mathrm{~kg} /$ cápita/día es sustancialmente mayor a los reportados en comunidades como X-Uilub, México (2.02 kg/capita/ día: Sánchez 1993), Sierra Leona (1.97 kg/capita/ día: Amoo-Gottfried y Hall 1999), sur de la India (1.9-2.2 kg/capita/día: Reddy 1981 y Hedge 1984, en: Bhatt y Sachan 2004), oeste de los Himalayas con (1.49 kg/capita/día: Bhatt et al. 1994, en: Bhatt \& Sachan, 2004) y sureste asiático (1.7-2.5 
kg/capita/día: Donovan 1981, en: Bhatt \& Sachan 2004), sin embargo es más bajo en relación con comunidades del noreste de la India (3.1-10.4 kg/capita/día: Maikhuri 1991 y Shankar 2000, en: Bhatt $\&$ Sachan 2004). Como el estudio se limitó a una época específica del año, es recomendable que se haga sobre un tiempo más prolongado que permita hacer una inferencia más acorde al ciclo anual de producción campesina y sobre una muestra más representativa.

Según Sánchez (1993), las familias grandes podrían cubrir las mismas necesidades básicas con el mismo combustible que las pequeñas, como se verificó en este estudio, aunque esto puede no ser totalmente cierto en algunas épocas para la comunidad de San José, ya que el número de personas que comen semanalmente en una vivienda aumenta o disminuye con la presencia o ausencia de obreros, con las implicaciones que esto tiene para el consumo, pues significa cambios en el número de comidas y el tipo de alimentos a preparar diariamente, como se constató con un aumento en el consumo según el numero de veces que se prende el fogón durante el día. De esta manera una familia de tres integrantes, dependiendo de la época del año y la contratación de obreros, puede convertirse en una familia de 5 integrantes con las implicaciones sobre consumo per cápita que esto tiene.

Existen varios factores que pueden explicar el alto consumo reportado, como la preparación de comida para los obreros que realizan actividades en la finca, ya que ésta hace parte del pago a cambio del trabajo realizado por estas personas y que en ocasiones suele servirse cuatro veces al día. En segundo lugar, el tipo y la cantidad de comidas que se preparan para el consumo humano y de los animales, pues hay alimentos que exigen de mucha energía para su preparación, como la yuca, el bore y el maíz. Otro factor es la época de recolección de información jun-ago, que es de siembra y cosecha de varios productos, lo que puede influir en el promedio anual, pues no se tuvieron en cuenta otras épocas en las que el consumo podría ser menor. Un cuarto factor podría ser el tiempo que dura el fogón encendido mientras no se preparaban alimentos, que en algunos casos llega a ser toda la noche. La suma de estos factores se refleja en la frecuencia diaria y el tiempo de encendido del fogón.

Cuando hay abundancia de un recurso es posible que éste sea aprovechado sin límite de uso, lo que hace que las personas gasten más de lo necesario para cubrir sus necesidades; tal es el caso de hogares que permitían que el fogón permaneciera prendido durante la noche o en horas del día sin estar necesariamente cocinando, lo que puede parecer un despilfarro, sin embargo esto puede explicarse bajo el supuesto de que el hogar tiene fácil acceso al recurso. Otras familias solían ser muy ahorrativas en la utilización del recurso pues no tenían acceso a fincas o bosques con buena disponibilidad de leña, por eso posiblemente se veían obligadas a hacer un uso más racional del recurso. Esta relación disponibilidad/demanda frecuentemente se ha abordado en otros estudios de leña, por ello se recomienda en futuras investigaciones tenerla en cuenta, ya que es de gran importancia para la planeación y el manejo de los recursos dendroenergéticos de una región.

La preferencia por la leña frente a otras fuentes de energía se explica porque presenta en la región fácil acceso y bajo costo, no sólo económico sino en cuanto a la facilidad de recolección, pues la mayoría de hogares no recorre grandes distancias para obtenerla y generalmente se obtiene de la propia finca. La distancia al sitio de recolección está condicionada por el acceso a las fuentes de obtención de leña, es decir que de acuerdo al tipo de actividades agrícolas y al lugar donde se desarrollan el campesino extrae la leña según acuerdo con el dueño de la tierra -ya se reportó anteriormente cómo el $63.5 \%$ de las propiedades son minifundios (0.5-5 ha).

\section{CONCLUSIONES}

En los casos en que no se tiene acceso a la tierra, ya sea por propiedad o por falta de trabajo, se deben recorrer distancias mayores para la obtención de leña. Todas las familias que se desplazan distancias mayores a $1 \mathrm{~km}$ la obtienen de palos caídos y secos ya sea en bordes de caminos, quebradas, potreros o cedida por otros propietarios. También recorren grandes distancias los recolectores en 
áreas de bosque poco intervenido o en regeneración (rastrojeras), respecto a las cuales las viviendas se encuentran muy lejos; sin embargo, en estos casos se hace con ayuda de un animal de carga. Según Bhatt y Sachan (2004), el deterioro del estado de la biomasa en los diferentes agroecosistemas incide en el aumento del tiempo y las distancias que los recolectores deben emplear para obtener el recurso; en el caso de San José de Suaita depende más del acceso a las fuentes, pues la mayoría obtiene la leña a una distancia relativamente corta. Sánchez (1993) afirma que la distancia a la cual las personas deben desplazarse para conseguir el recurso y el tipo de transporte condiciona la cantidad de leña que pueden recolectar. En San José de Suaita no se estableció la relación distancia/cantidad de leña recolectada, sin embargo, la cantidad de esta última sí puede variar según el recolector, ya sea hombre, mujer o niño, y si dispone de un animal de carga; también puede variar la cantidad que necesite llevar en el momento, es decir, si es para el día, para una preparación o para la semana. Aún así en San José de Suaita el consumo no está condicionado por las distancias a recorrer, pues el campesino busca la leña que necesita y la obtiene aún teniendo que recorrer largas distancias, aunque este caso se da en familias de muy bajos recursos y con poco acceso a la tierra.

La franja altitudinal en la que se encuentra San José de Suaita presenta un alto grado de deforestación para el país. Actualmente, para la región, los bosques de la meseta, con aproximadamente 300 ha, constituyen la reserva más importante de germoplasma, hoy conservada y protegida por la Fundación San Cipriano. Aunque allí se encuentran los árboles más grandes, la comunidad campesina ha desarrollado prácticas culturales para cosechar la leña dentro de los agroecosistemas de la región, sin explotar el recurso del bosque.

La selección de las especies más convenientes para conservar el fuego se basa en el conocimiento que sobre ellas tiene la sociedad. Mediante los sistemas de nominación popular se reconoce cuáles son las especies que definitivamente no deben ser eliminadas de los agroecosistemas; más aún, se conocen métodos de corte que promueven el desarrollo de varios ejes o renuevos, que pasados algunos meses proveen de nueva materia prima. El manejo de las unidades campesinas y la transmisión oral del conocimiento, de padres a hijos, mantiene procesos que evitan incidir de manera negativa sobre los bosques más conservados.

La preocupación por la extracción de leña de los bosques primarios se disipa, esto basado en el hecho de que sólo se encontraron 3 especies extraídas de allí. Las especies energéticamente más importantes se extraen de bosques en regeneración y cafetales, lo que los perfila como agroecosistemas con potencial de manejo para asegurar el suministro de leña en el futuro. Los bosques primarios o secundarios en avanzado estado de recuperación, como los de La Meseta, actualmente parecen lejanos y de difícil acceso para la explotación de leña por la mayor parte de la población. Sin embargo, La Meseta y otras zonas de bosques distribuidas por la región, como cabeceras de cuencas con poca intervención, pueden correr peligro en el futuro por la ampliación de cultivos agrícolas y tierras para ganado, más que por la extracción de madera para leña.

Algunas prácticas agrícolas pueden afectar en mayor o menor grado los diferentes agroecosistemas. Las modificaciones se producen sobre las poblaciones vegetales y en muchos estudios se ha establecido que estas poblaciones casi siempre hacen parte de agroecosistemas, y por lo tanto son afectadas por decisiones agrícolas y prácticas que favorecen a especies cultivadas (véase Bye 1979, 1981; Ford 1981, Chacón \& Gleissman 1982, Alcorn 1984, en Alcorn 1995). Las formas de recolección observadas responden a diferentes necesidades frente al mismo recurso, pero varían en las cantidades recolectadas, los sitios, las épocas y frecuencias de extracción, así como el tipo de necesidad que está destinada a cubrir. El tipo de recolección I, con niveles de extracción altos, es poco frecuente pero tiene un gran impacto sobre los agroecosistemas. Este tipo de recolección está asociado frecuentemente a otras actividades agrícolas de gran impacto como la apertura de áreas de cultivo. $\mathrm{Su}$ sostenibilidad está muy ligada a las actividades agrícolas de la región y depende mucho del tipo de 
cultivos que se realicen así como del ordenamiento territorial que el municipio tenga dispuesto. El tipo III tiene un nivel de extracción intermedio pero su frecuencia de recolección es alta; como su destino es la venta a la población urbana, se convierte en una forma de extracción cuya sostenibilidad debe ser considerada pues no se sabe cuántas familias dependen de esta práctica y, como ya se demostró, la dependencia de la leña comprada de la población urbana es bien importante.

El tipo de recolección II, con niveles de extracción bajos, tiene una frecuencia de recolección alta, lo que implica que satisface necesidades diarias de leña. En él pueden aparecer indiferentemente recolectores con acceso o no a agroecosistemas como cafetales o varsales. Este es probablemente el tipo de recolección más sostenible de los tres reportados pues está asociado a prácticas de manejo insertas en la comunidad, que hacen que el recurso sea cosechado paulatinamente a través de diversas maneras y fuentes.

La selección de las especies dendroenergéticas durante la recolección está dada por la facilidad de recolección, el destino para el cual son cosechadas y sus propiedades como leña. Es así como algunas son seleccionadas por su alto valor como combustible y son destinadas para la venta. Vale decir que durante el estudio se encontró cómo una carga compuesta únicamente de macanillo esmeraldo, macanillo negro, guamo o arrayán puede costar más que una carga de cualquier otra especie.

Algunas prácticas como la labor de rocería y de limpieza de los cultivos son fundamentales en la elaboración de planes de manejo con la comunidad, pues es el momento cuando seleccionan los individuos juveniles que más tarde van a ser los árboles utilizados como leña. Otras prácticas que merecen atención son la recolección periódica de palos secos, el secamiento de árboles por el método de anillado y la reutilización de madera que primero se utiliza como postes de cerca y luego como leña.

\section{AGRADECIMIENTOS}

Expresamos nuestro agradecimiento a la población campesina de la Inspección de Policía de San José de Suaita, en el municipio de Suaita (Santander), por su permanente apoyo en el desarrollo de esta investigación. A la Fundación San Cipriano que impulsó y apoyó logísticamente la realización de este estudio. A la Universidad Nacional de Colombia, a través del Instituto de Ciencias Naturales, por su apoyo académico a través de la biblioteca "Armando Dugand" y el Herbario Nacional Colombiano (COL). Los resultados presentados aquí hacen parte del proyecto Valoración de la diversidad biológica en la Inspección de San José de Suaita, Suaita, Santander, adelantado por el segundo autor.

\section{REFERENCIAS BIBLIOGRÁFICAS}

Agamez, H., A. Amaya, G. Galvis, I. A. Gómez, J. C. González, J. G. López, L. A. Moreno, B. O. Murillo, S. Pardo, C. O. Salcedo, M. C. Valderrama \& C. R. Vera. 1997. Plan Integral de Desarrollo Municipal 1995-1997. Suaita, Santander. Tesis de posgrado. Universidad de Bogotá Jorge Tadeo Lozano. Bogotá. Pg. 257.

Alcorn, J. B. 1995. The Scope and Aims of Ethnobotany in a Developing World. En: R. E. Schultes \& S. von Reis (eds.). Ethnobotany: Evolution of a Discipline. Chapman and Hall, London, UK. Págs. 23-39.

Alexiades, M. (ed.) 1996. Selected Guidelines for Ethnobotanical Research: A Field Manual. The New York Botanical Garden. New York. Pg. 306.

Amoo-Gottfried, K. \& D. O. Hall. 1999. A Biomass Energy Flow Chart for Sierra Leone. Biomass and Bioenergy 16: 361-376.

Batth, B. P. \& M. S. Sachan. 2004. Firewood Consumption Pattern of Different Tribal Communities in Northeast India. Energy Policy: 1-6.

Departamento Nacional de Planeación. 1989. Plan de Acción Forestal para Colombia. Gobierno de Holanda, Gobierno Alemán, FAO, Gobierno de Colombia. Bogotá. Pg. 76. 
Gentry, A. 2001. Patrones de diversidad y composición florística en los bosques de las montañas tropicales. En: Kepele M. \& A. Brown (eds.). Bosques nublados neotropicales. Instituto Nacional de Biodiversidad (INBIO). San José. Págs 85-123.

Henderson, A., S. P. Churchill \& J. L. Luteyn. 1991. Neotropical Plant Diversity. Nature 351.

Holdridge, L. 1977. Mapa de formaciones vegetales. Ministerio de Hacienda y Crédito Público. Instituto Geográfico Agustín Codazzi, Subdirección Agrológica. Bogotá. Pg. 238.

IDEAM. 1998. Estudio de la precipitación en el departamento de Santander. IDEAM. Bucaramanga. Pg. 31.

IDEAM. 1998. Estudio de la temperatura en el departamento de Santander. IDEAM. Bucaramanga.

Instituto Geográfico Agustín Codazzi (IGAC). 1982. Santander: aspectos geográficos. IGAC. Bogotá. Pg. 127.
Martin, G. 2000. Etnobotánica: manual de métodos. Serie Pueblos y Plantas. Manuales de Conservación. Montevideo. Pg. 240.

Murillo, A., J. Betancur, J.L. Fernández, G. Galeano, F. González, L.C. Jiménez, E.L. Linares, C.I. Orozco, J. Uribe, S. Suárez, D. Giraldo, C. Parra, H. Dueñas \& M. Galeano. 2002. Flora de San José de Suaita, Colombia. Instituto de Ciencias Naturales, Universidad Nacional de Colombia. En Libro de Resumen del VIII Congreso Latinoamericano de Botánica. Pg. 359.

Sánchez, M. C. 1993. Uso y manejo de la leña en X-Ulilub, Yucatán. Universidad Autónoma de Yucatán. Etnoflora yucatanense 8: 13-97.

SIAC. Sistema de Información Ambiental de Colombia. 2002. Perfil del estado de los recursos naturales y del medio ambiente en Colombia, 2001. Tomo III. Minambiente, IDEAM, SINCHI, IIAP, Humboldt, Invemar Embajada de los Países Bajos. Bogotá. Pg. 595. 
Anexo 1. Listado de especies utilizadas como leña en San José de Suaita

\begin{tabular}{|c|c|c|c|c|c|c|c|c|c|c|c|c|c|c|}
\hline $\begin{array}{l}\text { : } \\
\frac{0}{0} \\
\text { 응 } \\
\stackrel{\circ}{z}\end{array}$ & 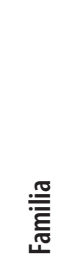 & Nombre científico & Nombre común & 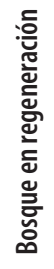 & $\begin{array}{l}\overline{\widetilde{J}} \\
\stackrel{\mathbb{J}}{0}\end{array}$ & 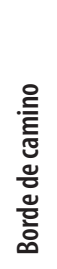 & $\begin{array}{l}\text { 일 } \\
\text { ¿ัँ }\end{array}$ & 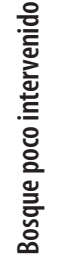 & 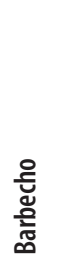 & 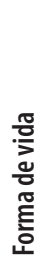 & 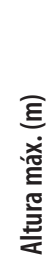 & 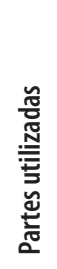 & 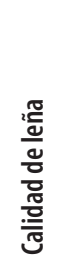 & 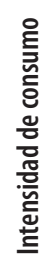 \\
\hline EV098 & ANNO & Guatteria sp. & Anón & * & & & & & & $A$ & 8 & $T, R$ & B & MB \\
\hline EV085 & APOC & Lacmellea edulis H. Karst) & Azulito & & & & & * & & $A$ & 2,5 & $T, R$ & B & MB \\
\hline EV137 & ARAL & Schefflera sphaerocoma (Benth) Harm. & Mano de león & * & & & & & & A & 3 & $\mathrm{~T}, \mathrm{R}$ & $\mathrm{R}$ & MB \\
\hline EV136 & ARAL & Schefflera karsteniana (March) Harm. & Mano de león & * & & & & & & $A$ & 3 & ni & $\mathrm{R}$ & MB \\
\hline EV047 & BRUN & Brunellia sp. & Guamo macho & & $*$ & & & & * & A & 5 & $\mathrm{~T}, \mathrm{R}$ & $\mathrm{E}$ & M \\
\hline \multirow[t]{3}{*}{ EV080 } & CAPR & Viburnum sp. & Juco & * & $*$ & * & & & & $A$ & 13 & $T, R$ & B & M \\
\hline & CECR & Cecropia Peltata L. & Orumo & * & $*$ & * & & & * & A & 11 & $\mathrm{R}$ & M & MB \\
\hline & CECR & Coussapoa villosa C.C.Berg \& P. Franco & Orumo & * & & * & & & * & $A$ & - & $\mathrm{R}$ & M & MB \\
\hline EV118 & CHLO & Hedyosmum racemosum (Ruiz \& Pav) G. Don & Granizo & * & & & & & & $A$ & 12 & $T, R$ & $\mathrm{R}$ & B \\
\hline EV106 & CLUS & $\begin{array}{l}\text { Clusia schomburgkiana (Planch. \& Triana) } \\
\text { Benth. ex Engl. }\end{array}$ & Gaque & * & & * & & & & $A$ & 10 & $\mathrm{~T}, \mathrm{R}$ & B & B \\
\hline EV107 & ERIC & Psammisia penduliflora (Dunal) Klotzsch & Camarón & & & * & & & & $a$ & 1 & $\mathrm{~T}, \mathrm{R}$ & B & B \\
\hline EV138 & EUPH & Acalypha macrostachya (Jacq.) & Mama juano & & & * & & & & $a$ & 3 & $\mathrm{~T}, \mathrm{R}$ & - & MB \\
\hline EV135 & EUPH & Alchornea grandis (Benth) & Hojiancho & * & & & & * & & $A$ & 10 & $\mathrm{~T}, \mathrm{R}$ & $R-M$ & MB \\
\hline EV090 & EUPH & Alchornea latifolia SW. & Uyamo & * & & & & * & & $A$ & 10 & $\mathrm{~T}, \mathrm{R}$ & $R-M$ & M \\
\hline EV139 & EUPH & Croton smithianus (Croisat) & Elemento & & & * & & & & $\mathrm{a}$ & 2 & $T, R$ & $\mathrm{R}$ & MB \\
\hline EV099 & EUPH & Indet. & Polvorín & * & & & & & & $A$ & 10 & $\mathrm{~T}, \mathrm{R}$ & B & B \\
\hline EV103 & HYPE & Vismia sp. 1 & Punta'e lanza & & & * & & & & A & 6 & $\mathrm{~T}, \mathrm{R}$ & B & MB \\
\hline EV039 & HYPE & Vismia sp. 2 & Manchador & * & & * & & & & A & 6 & $T, R$ & $\mathrm{E}$ & A \\
\hline EV126 & Indet & Indet. & Pescadito & * & & & & & & A & 5 & $\mathrm{~T}, \mathrm{R}$ & B & B \\
\hline EV046 & LAUR & Nectandra aff. laurel (Klotzsch ex. Nees) & Amarillo 1 & & $*$ & & & & * & A & 15 & $\mathrm{~T}, \mathrm{R}$ & - & M \\
\hline EV117 & LAUR & Persea caerulea (Ruiz \& Pav.) Mez & Curumacho & * & & * & & & & $A$ & 2 & $\mathrm{R}$ & - & MB \\
\hline EV142 & LYTH & Adenaria floribunda (Kunth) & Picurito & & & * & & & & $A$ & 4 & $T, R$ & - & B \\
\hline EV115 & MELA & Henriettella seemannii (Naudin) & Cubo'y hierro & * & & * & & & & $A$ & 4 & $\mathrm{~T}, \mathrm{R}$ & B & $A$ \\
\hline EV108 & MELA & Miconia cf. prasina (SW.) DC. & Macanillo blanco & * & & * & & & & $A$ & 5 & $T, R$ & B & M \\
\hline EV125 & MELA & Miconia cremophylla (Naudin) & Tuno blanco & * & & $*$ & * & & & $A$ & 2,5 & $\mathrm{~T}, \mathrm{R}$ & $\mathrm{R}$ & $A$ \\
\hline EV70 & MELA & Miconia dolichorrhyncha (Naudin) & $\begin{array}{l}\text { Macanillo esmeraldo, } \\
\text { Macanillo blanco }\end{array}$ & * & $*$ & & * & & * & A & 14 & $\mathrm{~T}, \mathrm{R}$ & $E$ & MA \\
\hline EV027 & MELA & Miconia elata (SW.) DC. & Macanillo negro & & & & * & & & A & 5 & $\mathrm{~T}, \mathrm{R}$ & $E$ & A \\
\hline EV076 & MELA & Miconia longifolia (Aubl.) DC. & Tuno negro & * & & * & & & & $A$ & 9 & $T, R$ & $\mathrm{R}$ & MB \\
\hline EV081 & MELA & Miconia minutiflora (Bonpl.) DC. & Tuno blanco & * & & * & * & & & $A$ & 8 & $T, R$ & $\mathrm{R}$ & A \\
\hline
\end{tabular}




\begin{tabular}{|c|c|c|c|c|c|c|c|c|c|c|c|c|c|c|}
\hline 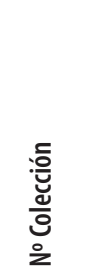 & 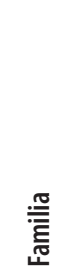 & Nombre científico & Nombre común & 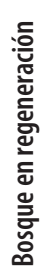 & 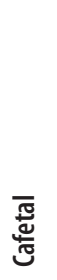 & 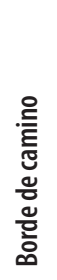 & $\begin{array}{l}\text { 일 } \\
\text { ¿ัँ }\end{array}$ & 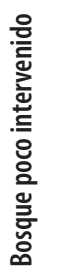 & $\begin{array}{l}\text { 읋 } \\
\text { 읋 } \\
\text { ळ }\end{array}$ & 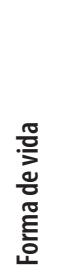 & 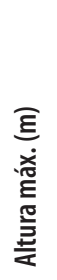 & 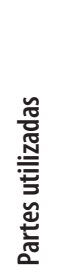 & 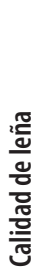 & 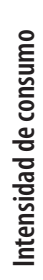 \\
\hline EV074 & MELA & Miconia serrulata (Naudin) DC. & Macanillo negro & & * & & & & $*$ & A & 7 & $T, R$ & $\mathrm{E}$ & $A$ \\
\hline EV120 & MELA & Miconia sp.1 & Macanillo Cenizo & & & * & & & & A & 3 & $T, R$ & M & B \\
\hline EV144 & MELI & Guarea guidonia (L.) (Sleumer) & Bailador & & * & * & & & $*$ & A & 15 & $T, R$ & $\mathrm{R}$ & M \\
\hline EV141 & MIMO & Albizia carbonaria (Britton) & Galapo & & * & & * & & & A & 20 & $T, R$ & $B$ & B \\
\hline EV045 & MIMO & Inga cf. ingoides (Rich) Willd. & Guamo santafereño* & & $*$ & & * & & $*$ & A & 13 & $T, R$ & $\mathrm{E}$ & MA \\
\hline EV088 & MIMO & Inga oerstediana (Benth) & Guamo torcido o común & & * & & * & & & A & 4 & $T, R$ & $\mathrm{E}$ & MA \\
\hline EV089 & MONI & Siparuna gesnerioides (Kunth) A. DC. & $\begin{array}{l}\text { Limoncito de monte, } \\
\text { limoncillo }\end{array}$ & * & & * & & & & a & 5 & $\mathrm{~T}, \mathrm{R}$ & $\mathrm{R}$ & MB \\
\hline EV130 & MORA & Ficus sp. & Lechero o Chivechi & * & * & & & & & A & 5 & $\mathrm{~T}, \mathrm{R}$ & M & $A$ \\
\hline EV072 & MYRS & Myrsine sp. & Cucharo & & & & & & $*$ & $A$ & 4 & $T, R$ & B & B \\
\hline \multirow[t]{2}{*}{ EV075 } & MYRT & Calycolpus moritzianus (0. Berg) Burret & Arrayán & * & & * & * & & & A & 9 & $T, R$ & $\mathrm{E}$ & B \\
\hline & MYRT & Indet. & Eucalipto & & & * & & & & A & 10 & $\mathrm{R}$ & B & MB \\
\hline \multirow[t]{2}{*}{ EV077 } & MYRT & Myrcia aff. fallax (Rich.) DC. & Payo & * & & * & & & & $A$ & 10 & $\mathrm{~T}, \mathrm{R}$ & B & A \\
\hline & MYRT & Psidium guajava (Rich.) DC. & Guayabo & & * & & * & & & A & 8 & $\mathrm{~T}, \mathrm{R}$ & B & M \\
\hline \multirow[t]{3}{*}{ EV073 } & MYRT & Psidium sp. & Menudito & & * & & & & * & $A$ & 9 & $\mathrm{~T}, \mathrm{R}$ & B & B \\
\hline & PINA & Pinus patula (Schltdl. \& Cham) & Pino & & & * & & & & A & 8 & $\mathrm{~T}, \mathrm{R}$ & $\mathrm{E}$ & M \\
\hline & POAC & Indet. & Guadua & & & & * & & & a & - & $\mathrm{T}, \mathrm{R}$ & - & MB \\
\hline \multirow[t]{2}{*}{ EV100 } & RUBI & Cinchona pubescens (Vahl) & Quino & & & * & & & & A & 6 & $\mathrm{~T}, \mathrm{R}$ & B & M \\
\hline & RUBI & Coffea arabiga var. Colombia & Café & & * & & & & & a & 3 & $\mathrm{~T}, \mathrm{R}$ & $\mathrm{R}$ & A \\
\hline EV069 & SAPO & Chrysophyllum sp. 1 & Caimo 0 caima* & & * & & & & & A & 4 & $T, R$ & B & B \\
\hline EV043 & SOLA & Cestrum cf. diversifolium (Francey) & Tinto & & * & & & & & $\mathrm{a}$ & 2 & $T, R$ & - & MB \\
\hline EV092 & THYM & Daphnopsis sp. & Aguancún & * & & & & & & $A$ & 2 & $\begin{array}{l}\mathrm{Rz}, \\
\mathrm{T}, \mathrm{R}\end{array}$ & B & M \\
\hline GG6059 & TILI & Heliocarpus americanus L. & Balso & & $*$ & & & & & A & 15 & $\mathrm{R}$ & $M$ & MB \\
\hline
\end{tabular}

Tipo de planta y parte utilizada: A (árbol), a (arbusto); T (tronco), R (ramas), Rz (raíz).

Calidad de leña: E (Exelente), B (Buena), R (Regular), M (Mala).

Nivel de consumo: Muy alto (MA), Alto (A), Medio-Alto (M-A), Medio (M), Muy Bajo (MB). 